\title{
Heritability of sexual proportion in experimental sex-ratio populations of Drosophila mediopunctata
}

\author{
FLAVIA ROQUE VARANDAS, MICHELLE CRISTINA SAMPAIO \& ANTONIO \\ BERNARDO CARVALHO* \\ Departamento de Genética, Instituto de Biologia, Universidade Federal do Rio de Janeiro, Caixa Postal 68011, CEP \\ 21944-970, Rio de Janeiro, Brazil
}

\begin{abstract}
Sex-linked meiotic drive genes are expected to spread quickly in populations and may cause their extinction because of the lack of one sex. Theoretically, the most general evolutionary response to these genes is the spread of autosomal suppressors of meiotic drive because of Fisher's Principle, a mechanism of natural selection that would correct uneven sexual proportions. Such adaptive response depends on heritable autosomal variation for sexual proportion, which seems to be lacking in most species with chromosomal sex-determination. Natural populations of Drosophila mediopunctata bear sex-ratio X chromosomes ('SR'), an X-Y meiotic drive system that leads to female bias. In this paper we show that sexual proportion is highly heritable $\left(h^{2}=41\right.$ per cent) in experimental populations of this species because of autosomal genes, thus fulfilling the conditions for adaptive evolution of sexual proportion. The spread of autosomal suppressors is expected to have a dual effect on sexual proportion, reducing both the female excess in the progenies of SR/Y males and the frequency of SR chromosomes. Hence, prior to the spread of their suppressors, SR chromosomes presumably attained a high frequency in natural populations of $D$. mediopunctata, causing a strong female bias.
\end{abstract}

Keywords: Drosophila mediopunctata, evolution of sexual proportion, Fisher's Principle, heritability, meiotic drive, sex-ratio.

\section{Introduction}

In several Drosophila species there is a trait known as 'sex-ratio': males carrying certain X chromosomes (generally called 'SR' and usually associated with chromosome inversions) produce progenies with a large excess of females because of X-Y meiotic drive (Gershenson, 1928; Jaenike, 1996). The X:2 and the X:21 gene arrangements of $D$. mediopunctata are SR chromosomes. However, several males carrying them do not produce female-biased progenies because the meiotic drive is partially suppressed by Y-linked (Carvalho \& Klaczko, 1994) and autosomal genes (see below). The progenies of wild-caught SR/Y males contain on average 21.8 per cent of males (Carvalho et al., 1989).

Normal X chromosomes ('ST') are transmitted by males to half of their progenies (the daughters), whereas SR ones achieve greater $\mathrm{X}$ transmission. Because of this meiotic drive advantage, SR

\footnotetext{
*Correspondence. E-mail: bernardo@acd.ufrj.br
}

chromosomes are expected to spread and become fixed, which might cause population extinction because of the rarity of males (Gershenson, 1928; Hamilton, 1967). However, stable SR polymorphisms are found in natural populations of Drosophila, as has been observed in D. pseudoobscura (Dobzhansky, 1958) and D. mediopunctata. In the latter species the frequency of SR remained around 13 per cent between 1987 and 1996 in the Itatiaia population (Carvalho et al., 1989; unpublished data). This stability implies some form of natural selection against SR, which has been found in $D$. pseudoobscura, the sole species investigated in this respect (Curtsinger \& Feldman, 1980; Beckenbach, 1996; and references cited therein). Thus, SR polymorphisms probably result from an equilibrium between meiotic drive and natural selection.

Another stabilizing mechanism may be provided by modifier genes that suppress meiotic drive; these genes 'rob' the meiotic drive advantage of SR, presumably preventing its fixation or leading to a lower equilibrium frequency (Stalker, 1961). Autoso- 
mal suppressors of sex-ratio are expected to evolve in SR-bearing populations because of 'Fisher's Principle' (Fisher, 1930; Hamilton, 1967; Wu, 1983; Bull \& Charnov, 1988), a mechanism of natural selection that would 'correct' uneven sex proportions as follows. In any sexually reproducing population half of the autosomal genes come from each sex, irrespective of its rarity. If there is a rare sex (such as males in SR-bearing populations), it will be effectively more fertile as a result of a greater per capita contribution to the next generation. Consequently, genes overproducing it (as autosomal suppressors of SR do) will spread, reducing gradually the unevenness of sexual proportion (and their own advantage). At the 1:1 equilibrium males and females are equally fertile and the sexual proportion becomes a neutral trait.

Autosomal suppressors of sex-ratio have been found in D. mediopunctata (Carvalho \& Klaczko, 1993), which seems to confirm that natural selection corrects biased sexual proportions caused by sex-chromosome meiotic drive. However, there are grounds to question the generality of this conclusion. First, autosomal suppressors of sex-ratio compatible with Fisher's Principle have been found only in $D$. mediopunctata; they have been unsuccessfully searched for in natural populations of $D$. pseudoobscura. In this species, the progenies of SR/Y males contain around 1 per cent of sons (Wu, 1983; and references cited therein). Furthermore, many attempts to detect genetic variation for sexual proportion in organisms with sex-chromosomes have failed (see Discussion), although it was present in other species with more labile sex-determining mechanisms such as arrhenotoky (Orzack \& Gladstone, 1994) and environmental sex determination (Bull et al., 1982). Of course, the sexual proportion cannot evolve if there is no genetic variation for it. The type of variation is also very relevant: Y-linked control of sexual proportion may lead to male bias, whereas female bias may result from cytoplasmic and X-linked control. Fisher's Principle - and hence, the 50 per cent equilibrium - applies strictly only to autosomal genes (Shaw, 1958; Hamilton, 1967; Bull \& Charnov, 1988; Frank, 1990). Finally, it should be noted that the evolutionary explanation for autosomal suppressors of sex-ratio relies entirely on Fisher's Principle. This theory is the most accepted explanation for the commonness of the 1:1 sexual proportion but it has undergone few direct tests (Bull \& Charnov, 1988; Conover \& Voorhees, 1990; Basolo, 1994), none of them in a system with clear autosomal variation for sexual proportion (Carvalho et al., in preparation).

(C) The Genetical Society of Great Britain, Heredity, 79, 104-112.
A more complete understanding of Fisher's Principle, autosomal suppressors of sex-ratio and their relationships requires studies on the heritability of the sexual proportion because the rate of approach to the 1:1 equilibrium depends on it; small heritabilities may lead to exceedingly slow correction of biased sexual proportions (Bulmer \& Bull, 1982). The objectives of this work were: (i) to measure the heritability of sexual proportion in sex-ratio populations of $D$. mediopunctata, without the interference of nonautosomal genes; (ii) to investigate the consequences (for the SR polymorphism) of the evolution of autosomal suppressors.

\section{Material and methods}

\section{Experimental SR populations}

Four homozygous SR populations were founded as shown in Fig. 1, carrying a single Y, cytoplasm and SR chromosome, and mixed autosomes. The ITA-24-P strain is the source of Y chromosomes for all populations. It was obtained from a pair-mating (hence, it contains a single $\mathrm{Y}$ chromosome) and carries an $\mathrm{X}$ chromosome (ST) that did not cause female bias (Carvalho et al., 1989). The wild-caught strains were collected at Parque Nacional do Itatiaia (State of Rio de Janeiro, Brazil) and were the source of most of the autosomal variation. Finally, the ITC-221-AN strain is the source of the X chromosomes for the SR populations. Its autosomal background is exactly the same as the ITA-24-P strain, because of more then 10 backcrosses. ITC-221-AN is homozygous for a single SR chromosome, obtained from a wild-caught male. Note that only the autosomes are genetically variable so, barring mutation, sex chromosomes could not interfere with the heritability measurement. The SR

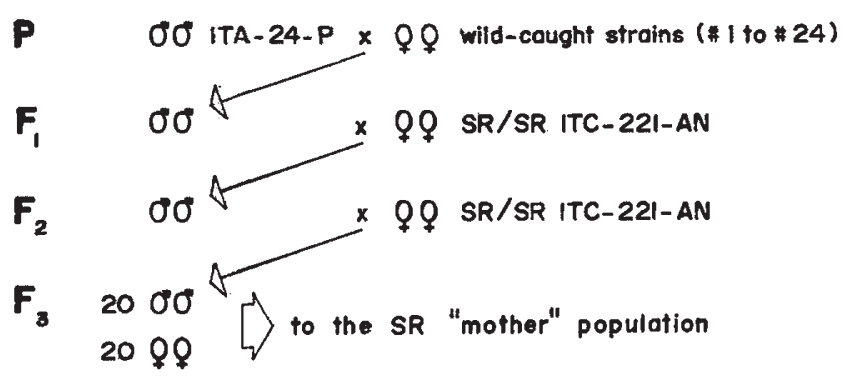

Fig. 1 Origin of the experimental SR populations. These procedures were repeated 24 times, with 24 different wildcaught isofemale strains. Thus, the SR 'mother' population was founded with 480 pairs. It was split two generations later into four populations (populations 1-4). 
chromosome employed $\left(21^{\mathrm{ITC}-221}\right)$ is unsuppressible by $\mathrm{Y}$ chromosomes and the $\mathrm{Y}$ chromosome $\left(\mathrm{Y}^{\mathrm{ITA}-24-\mathrm{P}}\right)$ is a nonsuppressor one (Carvalho \& Klaczko, 1994).

All populations were maintained in population cages under continuous generations, at $16.5^{\circ} \mathrm{C}$. The generation time was around five weeks.

\section{Measurement of SR expression}

We were interested in the heritability of sexual proportion which, in our experimental system, is the same as heritability of SR expression. Unless otherwise stated, SR expression was measured as follows. Each SR/Y male was aged for seven days and crossed with five virgin ITA-24-P females for six days. After this time flies were transferred to bottles to oviposit for 15 days, and were finally discarded. To reduce competition we added liquid ferment to the cultures regularly; the progenies produced were sexed and counted until bottle exhaustion. Only cultures producing at least 20 flies were considered. The result was always expressed as percentage males in the progeny.

\section{Father-son heritability}

Three hundred and forty adult males were collected from the SR populations and each one was crossed with five virgin females (SR/SR) from the same experimental population. Progenies were counted and up to three SR/Y sons were each crossed with five ITA-24-P females as described in the previous section. The SR expression of the sons (that is, the sexual proportion of their offspring) was averaged and regressed on their father's value. The heritability $\left(h^{2}\right)$ of the sexual proportion equals double the linear regression coefficient (Falconer, 1989). The experiment was carried out after 25 generations from the foundation of the experimental populations.

\section{Actual heritability}

Male age affects SR expression (Carvalho \& Klaczko, 1992). Thus, the ideal heritability estimate should be obtained by measuring fathers and sons under the normal life table of the species. Unfortunately, this could be carried out only for the fathers, because it is not possible to follow Drosophila families in populations. In the previous section we obtained a 'cross-environment' heritability (fathers with variable age, sons with fixed age); we applied the method developed by Lande (Riska et al., 1989) to the same data to place a lower bound on the actual heritability in the experimental populations, in which fathers and sons had a variable age. The method needs estimates of the cross-environment heritability, the phenotypic variance of the fathers (both obtained in the previous section) and the additive genetic variance under controlled-age conditions. The additive genetic variance estimate was provided by half-sib analysis (Falconer, 1989) of the families measured in the previous section, whose sons are mostly half-sibs. For this, we treated the three sons independently with a single classification ANOVA, using the fathers as the factor. This estimate of the additive variance was probably biased upwards because some sons were in fact full-sibs; this would result in a more conservative estimate of the actual $h^{2}$ (Riska et al., 1989).

\section{Sib analysis heritability}

Father--son estimates of heritability have the potential problem of Y chromosome effects (see Results). Sib analysis produces a mother ('dam') estimate of the heritability that is free from $\mathrm{Y}$ chromosome effects, because mothers do not carry or transmit this chromosome. To avoid the problem of few brothers in SR families, we carried out the sib analysis in two populations (populations 5 and 6) lacking female excess. These populations were founded at the same time and the same general procedure

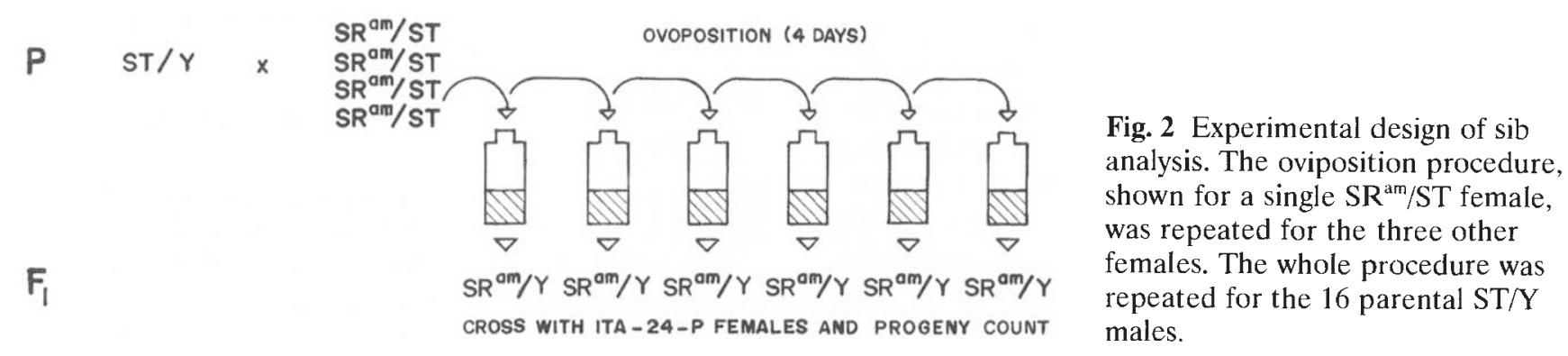

(c) The Genetical Society of Great Britain, Heredity, 79, 104-112. 
described for the SR ones was used, except that ITA-24-P females (instead of ITC-221-AN females) were used in the $F_{1}$ and $F_{2}$ crosses (see Fig. 1). Hence, these populations have exactly the same Y, cytoplasm and autosomal background as the SR populations, but they were homozygous for an $\mathrm{X}$ chromosome (ST) that did not cause female excess (Carvalho et al., 1989).

In the first step of the experiment we introgressed with six backcrosses a marked SR ( $\mathrm{SR}^{\text {am; }}$ yellow body) in the autosomal background of the ST populations and collected heterozygous $\mathrm{SR}^{\mathrm{am}} / \mathrm{ST}$ females. Then, 16 males from the ST populations were each crossed with four SR ${ }^{\text {am }} / \mathrm{ST}$ females (Fig. 2). These 64 females oviposited individually and were transferred six times (every four days) to new vials. We measured SR expression (as described before) in one $\mathrm{SR}^{\mathrm{am}} / \mathrm{Y}$ male from each vial, to avoid any commonenvironment effect. Heritabilities and their standard errors were calculated with a nested ANOVA, as described in Falconer (1989) and Becker (1992). Variance components were estimated using the restricted maximum likelihood method of SAS VARCOMP (SAS Institute, 1988).

\section{Statistical analysis}

All analyses were carried out with the untransformed proportion of males, which is an adequate scale for studies on Fisher's Principle (Carvalho \& Klaczko, 1993). The same results were obtained with the angular transformation (Sokal \& Rohlf, 1981). Unless otherwise stated, statistical analyses were performed using SYSTAT 5 (Wilkinson, 1992).

\section{Results}

\section{Phenotypic variance of sexual proportion}

Fig. 3 shows the distribution of the sexual proportion of the progenies of SR/Y fathers collected in the experimental populations. The large variance (205.3) suggests that sexual proportion may evolve quickly, for the Fisherian rate of evolution depends not only on the heritability but also on the phenotypic variance of the sexual proportion (Bulmer \& Bull, 1982). It is unavoidable that at least part of this variance was the result of binomial sampling error; this component does not respond to selection (Falconer, 1954). In order to reduce it, we have counted a large number of flies (on average, 110 flies per culture). The extra-binomial variance was significantly greater than zero (heterogeneity $\chi_{271}^{2}=3091, P<10^{-4}$ ) and may be estimated by the

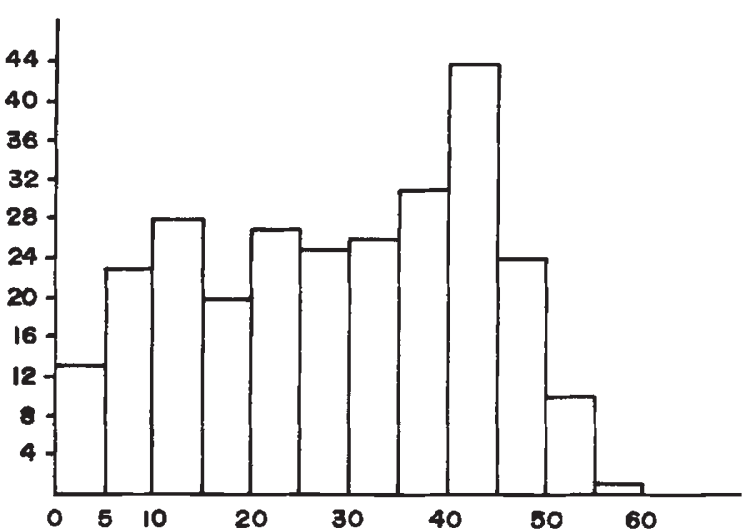

Fig. 3 Distribution of progenies of SR/Y males collected in the experimental SR populations $(n=272)$. Abscissapercentage males in the progeny; ordinate--absolute frequency of progenies.

simplified maximum likelihood method and by the chi-squared method (Robertson, 1951). Both methods yielded the same result: the extra-binomial component accounted for $\approx 87$ per cent of the total phenotypic variance of sexual proportion.

\section{Father-son heritability}

We have measured SR expression in 272 families (98000 flies counted). The heritability of sexual proportion was 41 per cent ( 95 per cent confidence interval: $22-60$ per cent), significant at the $10^{-4}$ level (Fig. 4). Mean SR expressions ( \pm SD) were $28.6 \pm 14.3$ per cent (fathers) and $28.2 \pm 15.5$ per cent (first sons). We have pooled all data because an analysis of covariance does not detect significant differences in the slope (that is, in the heritability) among the four SR experimental populations (not shown).

The method developed by Lande yielded a lower bound for the actual heritability of 30 per cent (additive variance under controlled age $=116.2$; $k=1.37$; see Riska et al., 1989). Although this value was based on quantities with a large error variance, it strongly suggests that the actual heritability was high. Thus, the sexual proportion is heritable not only in experiments with controlled age, but also under the normal life table of $D$. mediopunctata (at least under the life table in the experimental populations), in spite of the male-age effect on SR expression (Carvalho \& Klaczko, 1992). This result is also important because the experimental populations are being used in a test of Fisher's Principle (A. B. Carvalho, M. C. Sampaio, F. R. Varandas and L. B. Klaczko, unpublished observations); of course their 
expected rate of evolution is proportional to the actual heritability inside them.

\section{Sib analysis heritability}

We have measured the SR expression of $307 \mathrm{SR}^{\mathrm{am}} / \mathrm{Y}$ males in this experiment (25000 flies counted). As shown in Table 1 , mothers have a significant effect in the SR expression of their sons, which excludes the possibility that our previous $h^{2}$ estimate was caused solely by $\mathrm{Y}$ chromosome effects. The dam-component of heritability was $65 \pm 24$ per cent and the sire-component was $42 \pm 25$ per cent. The ANOVA was unbalanced because of failure of some crosses; thus, the standard errors should be taken as rough approximations (Becker, 1992).

Thus, we have three direct estimates of heritability. The father-son and the sire estimates are free

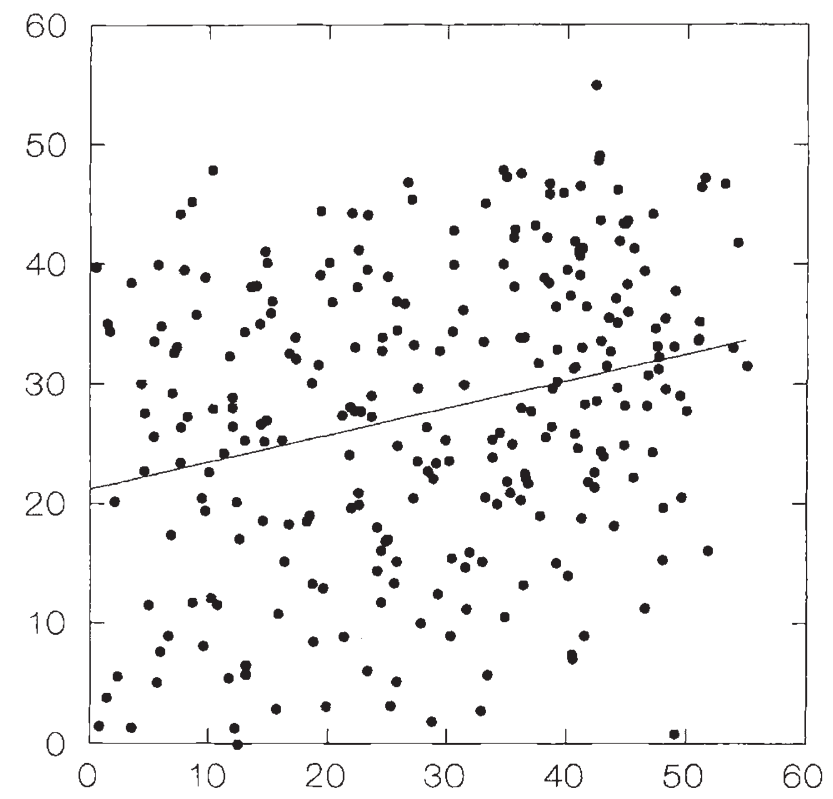

Fig. 4 Result of the father-son heritability experiment, showing the least squares regression line (slope $\pm \mathrm{SE}=$ $0.206 \pm 0.048$ ). Abscissa-percentage males in the progeny of fathers; ordinate-percentage males in the progeny of sons (average of up to three sons). from X, cytoplasmic and maternal effects, but they may be biased upwards by $\mathrm{Y}$ chromosome effects. The opposite applies to the dam estimate (except that $\mathrm{X}$ chromosome effects are unlikely because $\mathrm{SR}^{\mathrm{am}}$ was obtained few generations before the experiment). Autosomal genes are expected to have the same influence on the three estimates (indeed, they are the usual 'target' of heritability experiments), except for a possible upward bias in the mother-son estimate, because of dominance effects (Falconer, 1989). Because our three estimates are significant and reasonably close to each other (taking into account the usually large errors of $h^{2}$ estimates; Falconer, 1989), it can be safely concluded that they measured the net effect of autosomal genes on the sexual proportion. This result might have been expected because the experimental populations were founded with a single $\mathrm{Y}$ (and SR). However, a mutant suppressor $Y$ would spread very quickly (Wu, 1983) and would have caused an artefactual heritability.

As usual in genetical analysis, some caution is necessary concerning the generality of our results, for they are strictly valid only for the strains and experimental conditions we used (Falconer, 1989, p. 164).

\section{Discussion}

Sexual proportion is highly heritable in experimental sex-ratio populations of $D$. mediopunctata $\left(h^{2}=41\right.$ per cent), because of autosomal genes. Furthermore, there is a large extra-binomial phenotypic variance and the heritability remains high under the normal life table of the species, in spite of a male-age effect (Carvalho \& Klaczko, 1992). Thus, the sexual proportion has a large evolutionary potential in $D$. mediopunctata. These results complement and extend our previous study, which had detected at least four suppressor genes (one in each major autosome), with strong interactions among them (Carvalho \& Klaczko, 1993). To our knowledge, this is the first demonstration of autosomal heritable variation for sexual proportion in a species with

Table 1 Nested ANOVA of the sib analysis experiment

\begin{tabular}{lrcccrc}
\hline Source & & & \multicolumn{4}{c}{$\begin{array}{c}\text { Variance } \\
\text { components }\end{array}$} \\
\hline Sire & d.f. & MS & $F$ & $P$ & $h^{2} \pm \mathrm{SE}$ \\
Dam within sire & 15 & 302.18 & 2.28 & 0.016 & 9.55 & $42 \pm 25$ per cent \\
Error & 243 & 67.61 & 1.97 & $<0.001$ & 14.78 & $65 \pm 24$ per cent \\
\hline
\end{tabular}


sex-chromosomes. This type of variation is an essential condition for adaptive evolution of sexual proportion.

Our results showed that the sexual proportion of $D$. mediopunctata may evolve by natural selection (via Fisher's Principle) towards 50 per cent males. In fact, it evolved in the experimental populations from 16 per cent of males to 32 per cent in five years (49 generations); this rate is compatible with the heritability estimated in this work, which provides a clear experimental demonstration of Fisher's Principle (A. B. Carvalho, M. C. Sampaio, F. R. Varandas and L. B. Klaczko, unpublished observations). The question for the present paper is how this would apply to the natural populations of $D$. mediopunctata, and what would be the consequences for the SR polymorphism. The main difference between the natural populations and our experimental ones is that the former are not fixed for SR. Because these chromosomes probably are deleterious and depend on the meiotic drive for their maintenance, the spread of autosomal suppressors (because of Fisher's Principle) is expected to have a dual effect on the sexual proportion, reducing both the female bias produced by SR/Y males and the frequency of SR. The high heritability and phenotypic variance of sexual proportion in $D$. mediopunctata assures that this process may occur; given this and the commonness of suppression among wild-caught males (Carvalho et al., 1989), it is very probable that it had already been occurring since the origin of the autosomal suppressors. We propose that SR attained high frequencies when it first appeared in the species (because there were no suppressors that presently 'rob' its meiotic drive advantage), causing a very biased sexual proportion. Then the strong Fisherian selection caused the spread of autosomal suppressors which corrected the sexual proportion and caused a drop in the frequency of SR to its present level. The natural populations might now be near the equilibrium: the present Fisherian advantage of the suppressor alleles is small because the female bias is slight and because the suppressors are being selected during a minority of the time (only when present in SR/Y males, as they do not have detectable effects in ST/Y ones). A computer simulation of this process, using reasonable fitness values, is shown in Fig. 5. Although we do not have direct evidence that sex-linked meiotic drive had caused strong female bias in natural populations of $D$. mediopunctata, this has been documented in some populations of the butterfly Acraea encedon, whose sexual proportion dropped from nearly 50 per cent to 3 per cent between 1910 and 1965 because of this cause

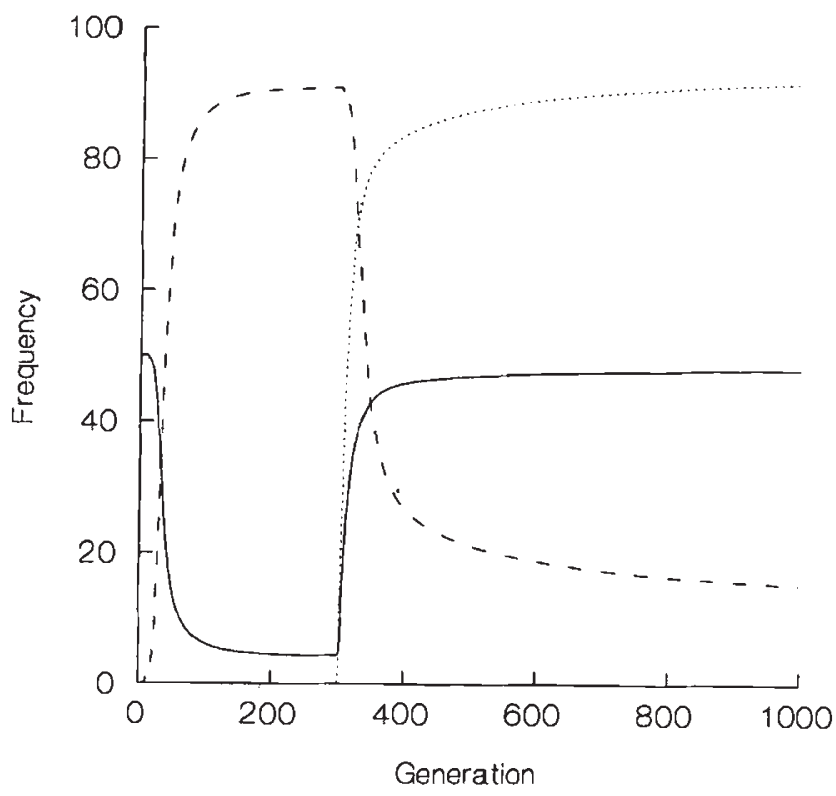

Fig. 5 Computer simulation of the fate of SR chromosomes (dashed line), their autosomal suppressors (dotted line) and the sexual proportion of the population (solid line). SR and a neutral autosomal suppressor $(s)$ were introduced at low frequency ( 1 per cent) in generations 10 . and 300 , respectively. SR/Y males with genotypes $S S, S s$ and ss produced progenies with 0 per cent, 25 per cent and 50 per cent of males, respectively. Fitness values (viability) were: ST/ST and ST/Y, 1.0; SR/ST, 1.1; SR/SR and $\mathrm{SR} / \mathrm{Y}, 0.85$. A stable equilibrium was attained after $\approx 2000$ generations. The equilibrium frequencies were: SR, 13 per cent; autosomal suppressor allele $(s), 94$ per cent.

(Chanter \& Owen, 1972). There is also good evidence that an X-linked meiotic drive gene, and its suppressors, became fixed in some natural populations of $D$. simulans (Merçot et al., 1995).

The above hypothesis can be treated quantitatively with two reasonable assumptions: (i) the SR polymorphism of $D$. mediopunctata is stable and results from an equilibrium between meiotic drive and some form of natural selection against SR; (ii) autosomal suppressors have evolved because of the spread of SR; prior to their evolution the meiotic drive was fully expressed (thus, in the past, $D$. mediopunctata would have been similar to $D$. pseudoobscura, whose SR/Y males produce almost only daughters). Edwards (1961) showed that the equilibrium frequency of SR under viability selection is:

$r e m=c \frac{a(1+2 c t)-2}{a(1+2 c t)-4 b c t}$,

where rem is the equilibrium gene ratio in males (the frequency of SR divided by the frequency of ST 
in adult males); $a, b$ and $c$ are the viabilities of $\mathrm{SR} / \mathrm{ST}, \mathrm{SR} / \mathrm{SR}$ and SR/Y, respectively (the viabilities of ST/Y and ST/ST are set to 1.0); and $t$ is the average proportion of females in the progeny of $\mathrm{SR} / \mathrm{Y}$ males. In the Itatiaia population, 13.2 per cent of the wild-caught males carried SR and they produced on average 21.8 per cent of males in their progenies (Carvalho et al., 1989; we have pooled X:2 and X:21 chromosomes); hence, rem was 0.154 and $t$ was 0.782 (and the sexual proportion of the population was around 46 per cent). Given these numbers and assumption (i), we may calculate $a, b$ and $c$ for any type of selection, such as selection in both sexes and SR recessive $(a=1 ; b=c)$; overdominance, etc. This is shown in the columns one to four of Table 2. Some cases are not considered because they could not explain the stable polymorphism of SR (selection restricted to $S R / Y$ males; see Curtsinger \& Feldman, 1980). Although we do not know which of the cases shown in Table 2 best represents the natural situation, one of them must do. Now, given these fitness values and assumption (ii), we may predict the frequency of SR prior to the evolution of autosomal suppressors, by setting $t=0.99$ in eqn (1). The result is shown in columns 5 and 6 of Table 2 : SR chromosomes were presumably much more frequent in the past, and may quite well have approached fixation; the female bias of the population would be, then, much stronger than its present level (46 per cent of males). Fecundity selection models (Curtsinger \& Feldman, 1980) produced essentially the same results (not shown).

The effect of binomial sampling error on the estimates of heritabilty of sexual proportion deserves some comments. This component inflates the phenotypic variance (Falconer, 1954) and, hence, reduces the heritability. If we had counted fewer flies per culture, we would probably had found a lower $h^{2}$ estimate. We simulated the effect of counting five flies per culture (instead of 110) in our father--son experiment and, in fact, the heritability dropped from 41 to 21 per cent. The reduction of heritability would be even larger in stable natural populations, in which the average lifetime progeny size is two. However, it is possible that the rate of evolution of sexual proportion would not be much affected, for it depends on the heritability and on the phenotypic variance (Bulmer \& Bull, 1982); the decrease in the former might be compensated by an increase in the latter. More theoretical work is needed to settle this point.

Previous studies have not detected genetic variation for sexual proportion in a variety of species with sex-chromosomes (Toro \& Charlesworth, 1982; and references cited therein; but see Frank, 1990). Curtsinger (1981) found a significant heritability (10 per cent) in non-SR strains of $D$. pseudoobscura, but this study did not exclude $\mathrm{X}$ - or Y-linked inheritance. The assumption of autosomal inheritance, usually held by standard quantitative genetic theory (Falconer, 1989), is critical for studies on sexual proportion because the evolutionary consequences are totally different in the case of $\mathrm{X}, \mathrm{Y}$ or cytoplasmic inheritance (Shaw, 1958; Hamilton, 1967; Bull \& Charnov, 1988; Frank, 1990). It is interesting to note that Curtsinger's study (with $D$. pseudoobscura) and Toro and Charlesworth's one (with $D$. melanogaster) used almost exactly the same experimental design with similar organisms, but obtained opposite results. The reasons for these differences between species are unknown.

The frequent occurrence of the 1:1 sexual proportion is usually attributed to Fisher's Principle, but this idea may be criticized (Williams, 1979; Toro \& Charlesworth, 1982) on the grounds that in species with sex-chromosomes, Fisher's Principle may be neither necessary (because Mendelian segregation already assures the 1:1 ratio), nor possible (because

Table 2 Predicted SR frequency and population sexual proportion before the evolution of the autosomal suppressors. Present values are 13 per cent and 46 per cent, respectively

\begin{tabular}{lcccccc}
\hline & \multicolumn{3}{c}{ Viabilities } & & \multicolumn{2}{c}{ Before suppression } \\
\cline { 2 - 4 } Type of selection & SR/ST $(a)$ & SR/SR $(b)$ & SR/Y $(c)$ & & $\%$ SR & $\%$ males \\
\hline SR recessive & 1 & 0.71 & 0.71 & & 42 & 29 \\
SR recessive & 1 & 0.90 & 0.66 & & 100 & 1 \\
Overdominance & 1.19 & 0.60 & 0.60 & & 23 & 39 \\
Overdominance & 1.19 & 1 & 0.52 & & 37 & 32 \\
Males unselected & 0.83 & 0.41 & 1 & & 36 & 32 \\
Males unselected & 0.90 & 0.10 & 1 & & 23 & 39 \\
\hline
\end{tabular}


genetic variation for sexual proportion seems to be absent). However, these species are prone to the invasion of meiotically driven sex-chromosomes that may cause strongly biased sexual proportions (Hamilton, 1967), which may be corrected only by mechanisms such as Fisher's Principle. In this paper we showed that the sexual proportion is highly heritable and variable in experimental populations of $D$. mediopunctata because of autosomal genes, thus fulfilling the conditions for its adaptive evolution. Given its large evolutionary potential (high heritability and large extra-binomial phenotypic variance) plus the rather high frequency of suppression among wild-caught SR/Y males (Carvalho et al., 1989), it is very unlikely that the adaptive process has just begun. Instead, we propose in this paper that the present state of the SR polymorphism (frequency of SR around 13 per cent; SR/Y males partially suppressed) represents a near equilibrium, after a past history of intense oscillation in gene frequencies and in sexual proportion. Thus, the Fisherian prediction that '... the sex-ratio will so adjust itself, under the influence of Natural Selection' (Fisher, 1930) may have found its full application in the past evolutionary history of species such as $D$. mediopunctata. We do not know whether this may be a common process because sex-linked meiotic drive is unknown in most species. However, it is worth noting that if the $D$. mediopunctata's SR were more deleterious, it might have been lost after the evolution of its suppressors, leaving no traces of its existence.

\section{Acknowledgements}

We thank C. Russo, B. C. Bitner-Mathé, A. SoléCava and two anonymous reviewers for valuable suggestions for the manuscript, and B. C. BitnerMathé for her help in the design of the experiments. We also thank Ms. Mônica Bahia Schlee for graphical assistance, Ms. Katia Coutinho and Ms. Renata Araújo for help in some experiments, and Ms. Cléa Knauer and Mr Silvio Nascimento for excellent technical assistance. This work was supported by Conselho Nacional de Desenvolvimento Científico e Tecnológico - CNPq, Coordenação de Aperfeiçoamento do Pessoal de Ensino Superior - CAPES, and Fundação Universitária José Bonifácio - FUJB.

\section{References}

BASOlO, A. L. 1994. The dynamics of Fisherian sex-ratio evolution: theoretical and experimental investigations. Am. Nat., 144, 473-490.
BECKENBACH, A. T. 1996. Selection and the "sex-ratio" polymorphism in natural populations of Drosophila pseudoobscura. Evolution, 50, 787-794.

BECKER, w. A. 1992. Manual of Quantitative Genetics, 5th edn. Academic Enterprises, Pullman, WA.

BULL, J. J. AND CHARNOV, E. L. 1988. How fundamental are Fisherian sex ratios? In: Harvey, P. H. \& Partridge, L. (eds) Oxford Surveys on Evolutionary Biology, vol. 5, pp. 96-135. Oxford University Press, Oxford.

BULL, J. J., VOGT, R. C. AND BULMER, M. G. 1982. Heritability of sex ratio in turtles with environmental sex determination. Evolution, 36, 333-341.

BULMER, M. G. AND BULL, J. J. 1982. Models of polygenic sex determination and sex ratio control. Evolution, 36, $13-26$.

CARvalho, A. B. AND KlaczKo, L. B. 1992. Age and sex-ratio expression in Drosophila mediopunctata. Genetica, 87, 107-111.

CARVAlho, A. B. AND KlaczKo, L. B. 1993. Autosomal suppressors of sex-ratio in Drosophila mediopunctata. Heredity, 71, 546-551.

CARVAlHO, A. B. AND KLACZKO, L. B. 1994. Y-linked suppressors of the sex-ratio trait in Drosophila mediopunctata. Heredity, 73, 573-579.

CARVAlho, A. B., PEIXOTO, A. A. AND KlaCZKo, L. B. 1989. Sex-ratio in Drosophila mediopunctata. Heredity, 62, 425-428.

CHANTER, D. O. AND OWEN, D. F. 1972. The inheritance and population genetics of sex ratio in the butterfly Acraea encedon. J. Zool. Lond., 166, 363-383.

CONOVER, D. O. AND VOORHEES, D. A. 1990. Evolution of a balanced sex ratio by frequency-dependent selection in a fish. Science, 250, 1556-1558.

Curtsinger, J. w. 1981. Artificial selection on the sex ratio in Drosophila pseudoobscura. J. Hered., 72, $377-381$.

CURTSINGER, J. W. AND FELdMAN, M. w. 1980. Experimental and theoretical analysis of the 'sex-ratio' polymorphism in Drosophila pseudoobscura. Genetics, 94, 445-466.

DOBZHANSKY, TH. 1958. Genetics of natural populations. XXVII. The genetic changes in populations of Drosophila pseudoobscura in the American Southwest. Evolution, 12, 385-401.

EDWARDS, A. W. F. 1961. The population genetics of 'sex-ratio' in Drosophila pseudoobscura. Heredity, 16, 291-304.

FALCONER, D. S. 1954. Selection for sex ratio in mice and Drosophila. Am. Nat., 88, 385-397.

FAlCONER, D. S. 1989. Introduction to Quantitative Genetics, 3rd edn. Longman, London.

F1SHER, R. A. 1930. The Genetical Theory of Natural Selection. Clarendon Press, Oxford.

FRANK, S. A. 1990. Sex allocation theory for birds and mammals. Ann. Rev. Ecol. Syst., 21, 13-55.

GERSHENSON, S. 1928. A new sex-ratio abnormality in Drosophila obscura. Genetics, 13, 488-507.

Hamilton, w. D. 1967. Extraordinary sex ratios. Science, 156, $477-488$ 
JAEniKe, J. 1996. Sex ratio meiotic drive in the Drosophila quinaria group. Am. Nat., 148, 237-254.

MERÇOT, H., ATLAN, A., JACQUES, M. AND MONTCHAMPmoreau, C. 1995. Sex-ratio distortion in Drosophila simulans: co-occurrence of a meiotic drive and a suppressor of drive. J. Evol. Biol., 8, 283-300.

ORZACK, s. H. AND GLADSTONE, J. 1994. Quantitative genetics of sex ratio traits in the parasitic wasp, Nasonia vitripennis. Genetics, 137, 211-220.

RISKA, B., PROUT, T. AND TURELLI, M. 1989. Laboratory estimates of heritabilities and genetic correlations in nature. Genetics, 123, 865-871.

ROBERTSON, A. 1951. The analysis of heterogeneity in the binomial distribution. Ann. Eugen., 16, 1-15.

SAS INSTITUTE 1988. SAS/STAT User's Guide, version 6.03. SAS Institute, Cary, NC.

SHAW, R. F. 1958. The theoretical genetics of the sex ratio.
Genetics, 43, 149-163.

SOKAL, R. R. AND ROHLF, F. J. 1981. Biometry, 2nd edn. W. H. Freeman and Company, New York.

S1ALKER, H. D. 1961. The genetic systems modifying meiotic drive in Drosophila paramelanica. Genetics, 46, 177-202.

TORO, M. A. AND CHARLESWORTH, B. 1982. An attempt to detect genetic variation in sex-ratio in Drosophila melanogaster. Heredity, 49, 199-209.

WILKINSON, L. 1992. SYSTAT for Windows, version 5. SYSTAT Inc., Evanston, IL.

WILliams, G. C. 1979. The question of adaptive sex ratio in outcrossed vertebrates. Proc. R. Soc., 205, 567-580.

wu, c.-1. 1983. The fate of autosomal modifiers of the sex-ratio trait in Drosophila and other sex-linked meiotic drive systems. Theor. Pop. Biol., 24, 107-120. 